\title{
Development of a Needs-Based Essential Care Service Package for Institutional Dementia Care in China
}

\author{
Tongda Sun ${ }^{1}$, Hongdao Meng ${ }^{2}$, Mingze Zhu ${ }^{3}$, Xiaoxin Dong ${ }^{1}$, Naidan Tu ${ }^{4}$, Ning Sun ${ }^{1}$, Rangcheng Jia ${ }^{1, *}$ \\ 1 Institute of Health Service Research, Ningbo College of Health Sciences, Ningbo 315100, Zhejiang Province, \\ China; suntongda@hotmail.com (T.S.); dongxiaoxin918@163.com (X.D.); sunning_ly@126.com (N.S.); jia- \\ rangcheng_66@163.com (R.J.) \\ 2 School of Aging Studies, University of South Florida, Tampa, FL33612, USA; meng@usf.edu \\ 3 College of Public Health, University of South Florida, Tampa, FL33612, USA; mingzezhu@usf.edu \\ 4 College of Arts and Sciences, University of South Florida, Tampa, FL33620, USA; naidantu@usf.edu \\ * Correspondence: jiarangcheng_66@163.com; Tel: +86-574-88112057
}

\begin{abstract}
Alzheimer's disease and related dementias (ADRD) remain a public health challenge in developing counties. We developed a needs-based essential care service package (ECSP) for care planning of persons living with dementia (PLWD) using a cross-sectional survey among PLWD in institutions in six cities in China $(n=1,299)$. Face-to-face interviews were conducted with caregivers of PLWD by trained staff between 2018 and 2019. Care service needs and utilization by the level of cognitive impairment were summarized. The average age of PLWD was 80.7 years. $76 \%$ of participants had severe cognitive impairment. The needs-based ECSP with 30 service items would be sufficient in supporting care services of PLWD in China, of which seven items are core care. The selection plan for ECSP at different levels is designed as "General Care Services + Selective Care Services", in which service items for low-, mid-and high-level care for PLWD are 7+3, 7+6, and $7+10$, respectively. The findings provide the first large-scale data on service needs and utilization of PLWD in mainland China. The ECSP for PLWD advanced in the paper was a practicable and effective quantitative management means. It is deserved to application in a large scale.
\end{abstract}

Keywords: Elderly with dementia; needs; utilization; essential care service package; long-term care system; health policy

\section{Introduction}

The prevalence of dementia has reached epidemic proportions worldwide, with 46.8 million individuals affected worldwide in 2015 and the number is projected to further increase to 74.7 million by 2030 and 131.5 million by 2050. The corresponding total medical cost associated with dementia care was estimated at USD 818 billion and it is expected to more than double in ten years [1,2]. In China, the prevalence of dementia among people aged 60 years and over was projected to increase from $5.8 \%$ in 2020 to $6.7 \%$ in 2030, with cases reaching 23.29 million in 2030 and the economic burden of dementia will increase from USD 167.74 billion in 2015 to USD 507.49 billion in 2030 [1-5]. Given the enormous toll that dementia will exert on individuals, families, and local/national governments, how to finance and deliver adequate care remains a public health and policy priorities for developed and developing countries alike.

In China, only $8.2 \%$ of the eligible population has purchased long-term care insurance (LTCI), resulting in continued challenges in meeting the needs of a large segment of the population, and especially for those living with dementia due to the often long and extended periods of disease progression and the associated functional decline [6-10]. One of the key public policy challenges in long-term care (LTC) financing is the balancing of two opposing forces: the need for adequate coverage at the individual/family level, and the required sustainability of funding [11]. Essential Service Package (ESP), a concept borrowed from management science in which a collection of must-have products and/or 
services is deemed essential for coverage and payment decisions. Proponents argue that ESP can improve equity, efficiency, and accountability in LTC financing. The concept of ESP has gained popularity in health and social insurance especially in developing countries where the value proposition for new policies is especially important. ESP recognizes and makes an explicit attempt to quantify the diverse needs of the population, which can contribute to the clarification of goals of providing adequate coverage while control cost increases, as few countries in the world can afford publicly financed universal coverage of all health and social services for their older adult population $[6,8,9,11]$.

In response to the rapid aging of its population and the associated challenges to the sustainability of the social security and pension system, the Chinese government has begun to establish a three-tiered universal LTC system as part of the 12th Five Year Plan [12]. The financing and delivery of dementia care has become a crucial component in LTC policy development as the rapidly increase in the number of PLWD. Since 2012, three cities (Shanghai, Qingdao, and Nanjing) have begun to implement pilot projects to integrate health insurance, LTCI, and a means-tested model [6,7,10]. These LTC demonstration projects have been expanded to a total of 15 cities by 2016 and plans for further expansion by 2020 [13,14]. All demonstration sites have implemented policy and administrative procedures based on local social and economic development levels [8-10]. Despite some early success, key challenges remain in terms of limited coverage of the population, dependence on local health insurance funds, lack of consistent client needs assessment and nursing standards, disconnect between service supply and need of long-term care services [6-10]. As a result, with the exception of six cities (Qingdao, Chengdu, Shanghai, Guangzhou, Suzhou, and Nantong), the expanded LTC coverage is still limited to older adults with severe disability and excluded PLWD [15].

Therefore, how to address the need from the rapid growth in the PLWD population in China under resource (financial and personnel) constrains has become an important area of research for LTC policy makers and researchers [16]. The objectives of this study were to describe the care service needs and utilization of PLWD in China, develop a needs-based ECSP; and to estimate the need for service provision by providers. The proposed ECSP can contribute to future research and policy development regarding the financing and delivery of health and social care for the growing PLWD population by informing the design elements for a universal healthcare coverage (UHC) of PLWD and an equity-based approach in dementia care, a key component of the evolving LTC system.

\section{Materials and Methods}

\subsection{Study design, setting, and population}

In China, the PLWD population is served in a wide range of settings: home, community, long-term care, rehabilitation, and psychiatry hospitals, as well as a limited number of nursing homes funded by the civil service system. We conducted a multi-city cross-sectional survey study to describe the health care and social service needs and their utilization in institutional settings. A two-stage stratified random sampling was used to select six cities of varying sizes and levels of economic development from diverse geographic provinces/administrative regions (Shanghai/east, Qingdao/northeast, Ningbo/east, Changzhou/central south, Chengdu/southwest, and Zhengzhou/central north) to participate in this study. From each city, dementia care facilities (nursing homes, geriatric hospitals, psychiatric hospitals, and other institutions) were then selected to participate in the study. Finally, eligible individuals in these sampling facilities were invited to complete the survey.

\subsection{Inclusion criteria}

Residents were eligible to be in the study if they 1) were 60 years or older, 2) had a family caregiver who is willing to provide written informed consent; 3) and meet one of the following diagnostic criteria for Alzheimer's disease and related dementia: (1) "The 
International Statistical Classification of Diseases and Related Health Problems" (10th revision) (coded as G30 Alzheimer's disease, F00 AD dementia, F01 vascular dementia, F02 dementia caused by other diseases classified elsewhere, F03 unspecified dementia) from the World Health Organization [17]. (2) "Diagnostic and Statistical Manual of Mental Disorders" from the American Psychiatric Association [18,19]. (3) Chinese Guidelines for Diagnosis and Treatment of Cognitive Impairment and Dementia [20,21]. Those who are less than 60 years old, undiagnosed, or unwilling to consent were excluded.

\subsection{Procedures}

All study personnel attended a five-day training program in which mock interviews were used to train the staff on how to consistently collect information using the questionnaire. Six teams of research staff then conducted the sampling and interviews in the city between July 2018 and October 2019. The sampling frame were all registered facilities licensed for dementia care based on local health service bureau and civil service administrative records. Upon selection into the sample, electronic medical record systems were queried to identify potentially eligible participants. Research staff then visited each facility and conducted face-to-face interviews caregivers (professional and/or family) regarding the sample persons and their care. Each interview lasted between 30 minutes and one hour.

\subsection{Measures}

The questionnaire and standard operating procedures were developed by reviewing the current literature and in consultation with dementia research experts. A structured questionnaire was developed which incorporated validated instruments to assess the individual and family characteristics and dementia care needs of participants included four domains: (1) demographics: age, gender, education, occupation, marital status, income, visit frequency, The Barthel Index score, mini-mental state exam (MMSE) levels; (2) health and functional status: type of dementia, clinical symptoms, comorbidities, medication, rehabilitation; and (3) perceived service needs and use.

The Barthel Index was used to assess the activities of daily living (ADL) of PLWD, which consists of ten items including bowels, bladder, grooming, toilet use, feeding, transfer, mobility, dressing, stairs, and bathing. The Barthel Index (0-100 points) was grouped into 5 levels: completely independent (100 points), mildly dependent (91-99 points), moderately dependent (61-90 points), severely dependent (21-60 points), and completely dependent (0-20 points) [22,23].

The Mini-Mental State Examination (MMSE) scale was used to assess the cognition severity of PLWD, consists of 7 categories, each category representing a different function: orientation to time, orientation to place, registration, attention and calculation, recall, language (naming, repetition, reading, and writing), and complex commands. The maximum score is 30 points. Two sets of cut-points were used to classify cognitive function based on their education level. For those with 6 years (elementary school) of education or less (PE): severe (0-9 points), moderate (10-15 points), mild (16-19 points), and normal (20-30 points). For those with more than 6 years of education (SE): severe (0-10 points), moderate (11-17 points), mild (18-23 points), and normal (24-30 points) [24,25].

\subsection{Essential care service package (ECSP)}

The ECSP is defined as a collection of health and social services necessary to care for PLWD in the care settings of home, community and institution, which including the type of service and hours of care needed for each type. The guiding principle for the development of the ECSP is to take person-centered care service need as the orientation, universal care coverage on safeguarding vulnerable populations as the focus, to balance the basic services needs for PLWD with affordability, equity, feasibility and sustainability in implementation by national and local governments [10,26,27]. There were three major steps involved in the development process: 1) estimation of perceived needs based on the 
service use data obtained from the survey; 2) estimation of time needed to provide each type of service; and 3) comparison between perceived needs and actual utilization data to determine which services should be included in the ECSP.

Informed by the Australian model of care and the ESP development for medical care, $80 \%$ of the health service needs were selected as the threshold for inclusion of the General Care Service of ECSP [26]. Thus, any service perceived by $80 \%$ or more of PLWD as being needed was included in the ECSP, so was any service with actual utilization rate of $60 \%$ or more were also included, that is, the basic care services for PLWD with low-level (mild cognitive impairment). Services that did not meet these criteria were deemed the Selective Care Service of ECSP for PLWD, that is, on the basis of the General Care Service, patients with mid-level (moderate cognitive impairment) and high-level (severe cognitive impairment) can choose additional service items. Based on the characteristics of special care services for PLWD, 30 basic service items were identified, which included activities of daily living (physical), medical/nursing/rehabilitation care, mental health care (psychological), and behavior management maintenance. The number services and their frequency of need/use are expected to increase for PLWD at mild, moderate, and severe stages of dementia and track the progression of the disease with increasing needs over time.

\subsection{Calculation of perceived demand, utilization rate and time spent on care services}

The formulas used for calculations are as follows: Perceived Needs $=\frac{\text { Numbers of subjects that responded YES to service demand (extremely }+ \text { much }+ \text { need })}{\text { Total surveyed subjects }} \times$ $100 \%$

Utilization Rate $=\frac{\text { Numbers of subjects that actually used the care service }}{\text { Total surveyed subjects }} \times 100 \%$

Average Service Time $=\frac{\text { Total time spent for all subjects who used the care service }}{\text { Total subjects who used the care service }} \times 100 \%$

\subsection{Estimation of the monthly service hours by the level of care}

\subsubsection{Calculation of the monthly nursing subsidy}

Based on the principals outlined in the Nursing Subsidy Policy for Workers' Compensation $[28,29]$ and the Implementation Policy for Long-Term Care Insurance in Chengdu (Demonstration) [30], three levels (low, mid, and high) were established to represent $30 \%, 40 \%$ and $50 \%$ of the average monthly wage of all urban employees in China in 2018. To facilitate interpretation and international comparison, all wage rates were converted into US dollars using the average annual exchange rate ( $1 \mathrm{USD}=6.6174 \mathrm{CNY})$ [31]. The governmental payment rate for the ECSP was set at $70 \%$ of the monthly nursing subsidy standard base rate at each corresponding level of care service [13,28].

\subsubsection{Calculation of total monthly serviced hours}

(1) Calculation of the total monthly serviced hours of low-level care based on the average care time of the General Care Service items in the ECSP.

Total Service Time Spent on Low - Level Care $=$ $\sum_{1}^{\mathrm{n}}$ (Service time for a general care service item in ECSP)

$(4)$

Where, $n$ is the number of the General Care Service items in the ECSP.

(2) Calculation of the total monthly service hours for mid-level care

Total Service Time Spent on Mid-Level Care =

Total Service Time Spent on Low-Level Care $\times\left(\frac{\text { Monthly subsidy quota for mid-level }}{\text { Monthly subsidy quota for low-level }}\right)$

(3) Calculation of the total monthly service hours for high-level care 
Total Service Time Spent on High-Level Care $=$

Total Service Time Spent on Low-Level Care $\times\left(\frac{\text { Monthly subsidy quota for high-level }}{\text { Monthly subsidy quota for low-level }}\right)$

\subsubsection{Calculation of total number of service items}

The total number of care service items is calculated from the total monthly service hours of each corresponding care level.

(1) Those care service items with perceived need greater than or equal to $80 \%$, or utilization rate of $60 \%$ or greater, obtained from sampled dementia patients will be determined as the General Care Service items in the ECSP, that is low-level care service.

(2) Calculation of the total number of mid-level care service items

Total number of mid-level service items $=$ Low-level service items $\times$ Total service time spent on mid-level care

Total service time spent on low-level care

(7)

(3) Calculation of the total number of high-level care service items

Total number of high-level service item $=$ Low-level service items $\times$ Total service time spent on high-level care

Total service time spent on low-level care

(8)

\subsection{Statistical Analysis}

All survey data were entered into EpiData database software with a double-entry method for data validation. Statistical analysis was performed using SPSS software version 26 (IBM Inc.). Continuous variables were described by Mean \pm Standard Deviation $(S D)$ with $\mathrm{t}$ and $\mathrm{F}$ tests used for significance testing. Categorical variables were described by percentages and $\chi^{2}$ test was used for significance testing. Two-tailed tests were used with the significance level set at $\alpha=0.05$. Since the service hours were not normally distributed, the natural logarithm transformation was used before analyses to alleviate the skewness of the data.

\section{Results}

\subsection{Sociodemographic and clinical characteristics of participants}

A total of 1573 questionnaires were distributed, and 1299 questionnaires were valid and included in the study, resulting in a response rate of $82.6 \%$. Table 1 shows that the average age is 80.7 years ( $S D=8.9$, range: $60-105$ years) and $59.0 \%$ were women. $54.8 \%$ of the respondents had less than 6 years of education. The majority of the respondents were widowed (53.2\%). Blue-collar workers, government officials, and farmers accounted for $32.3 \%, 21.7 \%$, and $19.9 \%$, of the employment status before disease onset, respectively.

Table 1. Sociodemographic characteristics of PLWD in care institutions

\begin{tabular}{|c|c|c|c|c|c|c|c|}
\hline \multirow[b]{2}{*}{ Variables } & \multirow{2}{*}{$\begin{array}{c}\text { Number of } \\
\text { caes }(\mathrm{n}, \% \\
)\end{array}$} & \multicolumn{4}{|c|}{ Levels of cognitive impairment according to MMSE } & \multirow[b]{2}{*}{$\chi^{2} / \mathrm{t}$-test } & \multirow[b]{2}{*}{ P-value } \\
\hline & & $\begin{array}{c}\text { Normal } \\
(\mathrm{n}=44)\end{array}$ & $\begin{array}{l}\text { Mild } \\
(\mathrm{n}=88)\end{array}$ & $\begin{array}{l}\text { Moderate } \\
(\mathrm{n}=213)\end{array}$ & $\begin{array}{l}\text { Severe } \\
(\mathrm{n}=954)\end{array}$ & & \\
\hline $\begin{array}{l}\text { Age, years, } \\
\text { Mean }(S D)\end{array}$ & $1299(100.0)$ & $75.0(7.9)$ & $77.7(9.2)$ & $79.0(8.7)$ & $81.6(8.8)$ & 15.147 & 0.000 \\
\hline Gender & & & & & & & \\
\hline Male & $533(41.0)$ & $20(3.8)$ & $41(7.7)$ & $95(17.8)$ & $377(70.7)$ & 3.505 & 0.320 \\
\hline Female & $766(59.0)$ & $24(3.1)$ & $47(6.1)$ & $118(15.4)$ & $577(75.3)$ & & \\
\hline Total & $\begin{array}{l}1299 \\
(100.0)\end{array}$ & $44(3.4)$ & $88(6.8)$ & $213(16.4)$ & $954(73.4)$ & & \\
\hline
\end{tabular}


Educational level

$\begin{array}{llllllll}\text { PE } & 712(54.8) & 34(4.8) & 48(6.8) & 131(18.4) & 498(69.9) & 5.958 & 0.114 \\ \text { SE } & 587(45.2) & 14(2.3) & 46(7.9) & 104(17.7) & 424(72.2) & \end{array}$

Occupation before retirement

\begin{tabular}{|c|c|c|c|c|c|c|c|}
\hline Blue-collar & 419 (32.3) & $10(2.3)$ & $26(6.1)$ & 73 (17.5) & $311(74.2)$ & 27.379 & 0.159 \\
\hline \multicolumn{8}{|l|}{ Worker } \\
\hline Farmer & $259(19.9)$ & $18(7.0)$ & $22(8.6)$ & $49(18.9)$ & $170(65.6)$ & & \\
\hline $\begin{array}{l}\text { White-collar } \\
\text { worker }\end{array}$ & $141(10.9)$ & $4(3.0)$ & $15(10.5)$ & $21(15.0)$ & $101 （ 71.4)$ & & \\
\hline $\begin{array}{l}\text { Government } \\
\text { official }\end{array}$ & $281 \quad(21.7)$ & $8(3.0)$ & $19(6.8)$ & $37(13.2)$ & $217(77.0)$ & & \\
\hline Unemployed & $40(3.1)$ & $3(7.9)$ & $1(2.6)$ & $7(18.4)$ & $29(71.1)$ & & \\
\hline Other & $158(12.1)$ & $2(1.4)$ & $10(6.1)$ & $31(19.5)$ & $116(73.2)$ & & \\
\hline \multicolumn{8}{|l|}{ Marital status } \\
\hline Partnered & $524(40.3)$ & $27(5.1)$ & $35(6.7)$ & $101 （ 19.2)$ & $361(69.0)$ & 19.779 & 0.071 \\
\hline Divorced & $28(2.1)$ & $1(3.7)$ & $2(7.4)$ & $8(29.6)$ & $16(59.3)$ & & \\
\hline Widowed & $691(53.2)$ & $17(2.4)$ & $48(7.0)$ & $95(13.7)$ & $532(77.0)$ & & \\
\hline Single & $56 \quad(4.3)$ & $1(1.84)$ & $3(5.44)$ & $10(18.21)$ & $42(74.51)$ & & \\
\hline \multicolumn{8}{|l|}{ type of dementia } \\
\hline $\mathrm{AD}$ & $702(54.0)$ & $21(3.0)$ & $59(8.4)$ & $142(20.2)$ & $480(68.4)$ & 40.193 & 0.000 \\
\hline VD & $430(33.1)$ & $12(2.9)$ & $12(2.9)$ & $41(9.5)$ & $364(84.6)$ & & \\
\hline Other & $167(12.9)$ & $7(4.4)$ & $13(7.5)$ & $24(14.5)$ & $123(73.6)$ & & \\
\hline \multicolumn{8}{|c|}{ No. of comorbidities } \\
\hline 0 & $61(4.7)$ & $1(1.6)$ & $4(6.6)$ & $11(18.0)$ & $45(73.8)$ & 38.070 & 0.034 \\
\hline 1 & $631(48.6)$ & $23(3.6)$ & $43(6.8)$ & $106(16.8)$ & $459(72.7)$ & & \\
\hline 2 & $316(24.3)$ & $10(3.2)$ & $19(6.0)$ & $42(13.3)$ & $245(77.5)$ & & \\
\hline 3 & $196(15.1)$ & $5(2.6)$ & $16(8.2)$ & $32(16.3)$ & $143(73.0)$ & & \\
\hline 4 & $70(5.4)$ & $2(2.9)$ & $5(7.1)$ & $18(25.7)$ & $45(64.3)$ & & \\
\hline 5 and above & $25 \quad(1.9)$ & $3(12.0)$ & $1(4.0)$ & $4(16.0)$ & $17 \quad(68.0)$ & & \\
\hline \multicolumn{8}{|l|}{ The Barthel Index } \\
\hline $\begin{array}{l}\text { Complete in- } \\
\text { dependence }\end{array}$ & $42(3.2)$ & $7(16.7)$ & $11(26.2)$ & $15(35.7)$ & $9(21.4)$ & 347.5 & 0.000 \\
\hline $\begin{array}{l}\text { Mild depend- } \\
\text { ence }\end{array}$ & $34(2.6)$ & $1(2.9)$ & $14(41.2)$ & 11 (32.4) & $8(23.5)$ & & \\
\hline
\end{tabular}




\begin{tabular}{|c|c|c|c|c|c|c|}
\hline $\begin{array}{l}\text { Moderate } \\
\text { pendence }\end{array}$ & De- & $189(14.5)$ & $12(6.3)$ & $26(13.8)$ & $58(30.7)$ & $93(49.2)$ \\
\hline $\begin{array}{l}\text { Heavy } \\
\text { pendence }\end{array}$ & de- & 311 (23.9) & $14(4.5)$ & $18(5.8)$ & $89(28.6)$ & $190(61.1)$ \\
\hline $\begin{array}{l}\text { Complete } \\
\text { pendence }\end{array}$ & de- & $723(55.7)$ & $10(1.4)$ & $19(2.6)$ & $40(5.5)$ & $654(90.5)$ \\
\hline
\end{tabular}

\subsection{Cognition impairment}

Table 1 shows that $73.4 \%$ of PLWD were rated as severe cognitive impairment, followed by moderate $(16.4 \%)$, and mild $(6.8 \%)$. Significant differences exist among different age groups, types of dementia, numbers of comorbidities, and ADL (the Barthel Index) $(\mathrm{P}<0.05)$. As age increases, moderate and severe cognitive impairment ratios also appear to increase. Besides, the proportion of severe cognitive impairment in vascular dementia (VD, $84.6 \%$ ) and other types of dementia $(73.6 \%)$ is higher than that of Alzheimer's disease (AD, 68.4\%). As the elder becomes more dependent upon others for ADL, their corresponding degree of cognitive impairment increases. The proportion of severe cognitive impairment has gradually increased from $21.4 \%$ of complete independence to $90.5 \%$ of total dependence. However, there was no significant difference in gender, education level, occupation, and marriage $(\mathrm{P}>0.05)$.

\subsection{Perceived Serivce Need}

Table 2 shows that seven services were reported as essential for PLWD (greater than or equal to $80 \%$ of respondents): primary care visit $(91.7 \%)$, medication management $(87.8 \%)$, regular health evaluation $(84.8 \%)$, medical records/care plan $(84.1 \%)$, using the toilet $(82.4 \%)$, housekeeping $(81.3 \%)$, and grooming $(81.0 \%)$. These services were included in the Essential Care Services for PLWD. Service needs in dining, grooming, using the toilet and expectoration care have a significant differences by the levels of cognitive impairment $(\mathrm{P}<0.05)$.

Table 2. Perceived service needs of PLWD in care institutions (\%)

\begin{tabular}{|c|c|c|c|c|c|c|c|}
\hline \multirow[b]{2}{*}{ Services } & \multicolumn{5}{|c|}{ Levels of cognitive impairment according to MMSE } & \multirow[b]{2}{*}{$\chi^{2}$} & \multirow[b]{2}{*}{ P-value } \\
\hline & $\begin{array}{l}\text { Normal } \\
(\mathrm{n}=44)\end{array}$ & $\begin{array}{l}\text { Mild } \\
(\mathrm{n}=88)\end{array}$ & $\begin{array}{l}\text { Moderate } \\
(\mathrm{n}=213)\end{array}$ & $\begin{array}{l}\text { Severe } \\
(\mathrm{n}=954 \\
)\end{array}$ & $\begin{array}{c}\text { Total } \\
(n=1299)\end{array}$ & & \\
\hline 1.Housekeeping & 76.3 & 74.7 & 86.4 & 81.0 & 81.3 & 6.59 & 0.086 \\
\hline 2.Transportation & 59.4 & 46.1 & 51.0 & 49.0 & 49.4 & 1.88 & 0.599 \\
\hline 3.Dining & 61.1 & 63.8 & 73.4 & 81.0 & 78.1 & 23.10 & 0.000 \\
\hline 4.Dressing/Grooming & 59.5 & 58.8 & 76.6 & 84.7 & 81.0 & 47.69 & 0.000 \\
\hline 5.Using the toilet & 52.8 & 54.4 & 71.9 & 88.3 & 82.4 & 102.00 & 0.000 \\
\hline $\begin{array}{l}\text { 6.Home } \\
\text { safety/modifications }\end{array}$ & 62.9 & 58.2 & 66.3 & 68.3 & 67.2 & 3.76 & 0.289 \\
\hline $\begin{array}{l}\text { 7.Community day } \\
\text { care }\end{array}$ & 63.6 & 51.9 & 50.0 & 47.4 & 48.6 & 3.97 & 0.264 \\
\hline $\begin{array}{l}\text { 8.Medical } \\
\text { records/Care plan }\end{array}$ & 79.4 & 88.3 & 80.2 & 84.8 & 84.1 & 3.97 & 0.265 \\
\hline 9.Primary care visit & 90.0 & 85.7 & 92.3 & 92.1 & 91.7 & 4.42 & 0.220 \\
\hline 10.Regular health & 83.3 & 87.2 & 81.0 & 85.5 & 84.8 & 2.95 & 0.400 \\
\hline
\end{tabular}




\begin{tabular}{|c|c|c|c|c|c|c|c|}
\hline $\begin{array}{r}\text { 11.Medication } \\
\text { management }\end{array}$ & 81.6 & 87.7 & 89.8 & 87.7 & 87.8 & 2.12 & 0.548 \\
\hline 12.Expectoration care & 48.5 & 57.1 & 56.8 & 65.9 & 63.3 & 10.56 & 0.014 \\
\hline 13.Oral care & 64.9 & 62.5 & 62.6 & 66.9 & 65.8 & 1.76 & 0.624 \\
\hline 14.Pain management & 50.0 & 53.8 & 54.6 & 61.7 & 59.6 & 6.11 & 0.106 \\
\hline $\begin{array}{l}\text { 15.Emergency } \\
\text { assistance }\end{array}$ & 58.3 & 68.4 & 65.5 & 70.2 & 68.9 & 3.63 & 0.305 \\
\hline $\begin{array}{l}\text { 16.Specialty nursing } \\
\text { care }\end{array}$ & 61.1 & 75.0 & 68.4 & 71.6 & 71.0 & 3.12 & 0.373 \\
\hline 17.Family guest beds & 38.7 & 46.1 & 40.3 & 44.5 & 43.8 & 1.57 & 0.667 \\
\hline 18.Companion & 62.2 & 68.8 & 65.1 & 71.0 & 69.6 & 3.62 & 0.306 \\
\hline 19.Life enrichment & 62.9 & 62.5 & 61.1 & 66.0 & 64.8 & 1.89 & 0.596 \\
\hline $\begin{array}{l}\text { 20.Healthcare } \\
\text { consultation and } \\
\text { education }\end{array}$ & 70.6 & 77.9 & 75.8 & 79.2 & 78.3 & 2.26 & 0.520 \\
\hline $\begin{array}{l}\text { 21.Mental health } \\
\text { counseling }\end{array}$ & 64.9 & 66.7 & 63.4 & 63.9 & 64.0 & 0.29 & 0.961 \\
\hline 22.Hospice care & 62.5 & 56.8 & 66.3 & 69.0 & 67.5 & 5.16 & 0.160 \\
\hline 23.Caregiver training & 63.6 & 66.2 & 66.5 & 65.8 & 65.8 & 0.11 & 0.991 \\
\hline 24.Respite care & 56.7 & 62.2 & 56.6 & 59.8 & 59.3 & 0.95 & 0.813 \\
\hline $\begin{array}{l}\text { 25.ADL training and } \\
\text { prevention of } \\
\text { anti-lost }\end{array}$ & 69.7 & 64.1 & 65.5 & 62.5 & 63.3 & 1.29 & 0.733 \\
\hline $\begin{array}{l}\text { 26.Medical } \\
\text { equipment } \\
\text { purchase, rental, } \\
\text { and usage guide }\end{array}$ & 47.1 & 48.0 & 42.8 & 50.2 & 48.8 & 3.46 & 0.326 \\
\hline $\begin{array}{l}\text { 27.Hazard prevention } \\
\text { and self-rescue }\end{array}$ & 58.8 & 63.6 & 57.5 & 65.5 & 63.9 & 4.58 & 0.205 \\
\hline $\begin{array}{l}\text { 28.Legal inquiry } \\
\text { assistance }\end{array}$ & 35.5 & 45.3 & 46.3 & 52.2 & 50.3 & 5.73 & 0.126 \\
\hline $\begin{array}{c}\text { 29.Pressure ulcer } \\
\text { prevention }\end{array}$ & 56.8 & 65.4 & 64.5 & 68.9 & 67.6 & 3.76 & 0.289 \\
\hline 30.Pairing assistance & 42.4 & 44.2 & 43.5 & 44.6 & 44.3 & 0.12 & 0.989 \\
\hline
\end{tabular}

\subsection{Service Use}

Table 3 shows that ten services with utilization rates greater than or equal to $60 \%$ : primary care visit $(83.8 \%)$, regular health evaluation $(78.8 \%)$, medical records/care plan $(77.2 \%)$, medication management $(76.1 \%)$, using the toilet $(76.1 \%)$, dining $(72.7 \%)$, dressing/grooming $(72.5 \%)$, healthcare consultation and education $(67.4 \%)$, housekeeping $(66.5 \%)$, and pressure ulcer prevention $(60.4 \%)$. Among them, the rate of the need of dining, healthcare consultation and education, pressure ulcer prevention was lower than $80 \%(78.1 \%, 78.3 \%$, and $67.6 \%$, respectively), and the rates of the need of the other seven services was higher than $80 \%$ (Table 2). The above ten service items were also included in 
the ECSP for PLWD with low-level care (mild cognitive impairment), and the remaining twenty items were included in the optional items of the ECSP for PLWD with medium and high-level care (moderate and severe cognitive impairment). The utilization rate of dining, grooming and using the toilet have significant difference with the levels of cognitive impairment $(\mathrm{P}<0.05)$

Table 3. Service Utilization of PLWD in care institutions (\%)

\begin{tabular}{|c|c|c|c|c|c|c|c|}
\hline \multirow{3}{*}{ Services } & \multicolumn{5}{|c|}{ Levels of cognitive impairment according to MMSE } & \multirow{3}{*}{$\chi^{2}$} & \multirow{3}{*}{ P-value } \\
\hline & $\begin{array}{c}\text { Normal } \\
(\mathrm{n}=44)\end{array}$ & $\begin{array}{l}\text { Mild } \\
(\mathrm{n}=88)\end{array}$ & $\begin{array}{l}\text { Moderate } \\
(\mathrm{n}=213)\end{array}$ & $\begin{array}{l}\text { Severe } \\
(\mathrm{n}=954\end{array}$ & $\begin{array}{c}\text { Total } \\
(\mathrm{n}=1299)\end{array}$ & & \\
\hline & & & & ) & & & \\
\hline 1.Housekeeping & 66.7 & 63.5 & 74.6 & 64.8 & 66.5 & 7.30 & 0.063 \\
\hline 2.Transportation & 36.7 & 29.2 & 37.6 & 27.9 & 29.9 & 7.43 & 0.059 \\
\hline 3.Dining & 62.5 & 56.8 & 68.9 & 75.4 & 72.7 & 15.53 & 0.001 \\
\hline 4.Dressing/Grooming & 53.1 & 45.1 & 71.2 & 75.8 & 72.5 & 37.52 & 0.000 \\
\hline 5.Using the toilet & 46.7 & 50.7 & 67.4 & 81.2 & 76.1 & 57.73 & 0.000 \\
\hline $\begin{array}{l}\text { 6.Home } \\
\text { safety/modifications }\end{array}$ & 50.0 & 43.5 & 55.4 & 51.7 & 51.8 & 2.93 & 0.403 \\
\hline $\begin{array}{l}\text { 7.Community day } \\
\text { care }\end{array}$ & 45.5 & 37.5 & 36.2 & 30.5 & 32.4 & 5.95 & 0.114 \\
\hline $\begin{array}{l}\text { 8.Medical } \\
\text { records/Care plan }\end{array}$ & 78.6 & 83.3 & 75.9 & 76.9 & 77.2 & 1.77 & 0.622 \\
\hline 9.Primary care visit & 77.8 & 78.7 & 87.9 & 83.6 & 83.8 & 4.97 & 0.174 \\
\hline $\begin{array}{l}\text { 10.Regular health } \\
\text { evaluation }\end{array}$ & 85.7 & 83.3 & 77.2 & 78.5 & 78.8 & 1.98 & 0.577 \\
\hline $\begin{array}{r}\text { 11.Medication } \\
\text { management }\end{array}$ & 66.7 & 76.3 & 81.3 & 75.2 & 76.1 & 4.93 & 0.177 \\
\hline 12.Expectoration care & 60.0 & 53.5 & 48.3 & 47.4 & 48.3 & 2.68 & 0.444 \\
\hline 13.Oral care & 58.1 & 57.1 & 55.8 & 58.4 & 57.9 & 0.40 & 0.940 \\
\hline 14.Pain management & 46.7 & 37.1 & 40.7 & 46.3 & 44.8 & 3.58 & 0.311 \\
\hline $\begin{array}{l}\text { 15.Emergency } \\
\text { assistance }\end{array}$ & 50.0 & 44.3 & 45.0 & 46.6 & 46.3 & 0.42 & 0.937 \\
\hline $\begin{array}{l}\text { 16.Specialty nursing } \\
\text { care }\end{array}$ & 51.7 & 58.3 & 53.8 & 52.1 & 52.8 & 1.14 & 0.767 \\
\hline 17.Family guest beds & 26.9 & 31.4 & 29.8 & 30.1 & 30.1 & 0.19 & 0.979 \\
\hline 18.Companion & 48.0 & 42.9 & 42.0 & 40.2 & 40.9 & 0.84 & 0.840 \\
\hline 19.Life enrichment & 37.5 & 40.6 & 39.3 & 34.6 & 35.9 & 1.93 & 0.587 \\
\hline $\begin{array}{l}\text { 20.Healthcare } \\
\text { consultation and } \\
\text { education }\end{array}$ & 78.6 & 69.0 & 68.5 & 66.5 & 67.4 & 2.02 & 0.569 \\
\hline $\begin{array}{l}\text { 21.Mental health } \\
\text { counseling }\end{array}$ & 63.0 & 55.6 & 56.5 & 49.7 & 51.6 & 4.62 & 0.201 \\
\hline 22.Hospice care & 40.0 & 28.6 & 32.2 & 29.5 & 30.1 & 1.67 & 0.645 \\
\hline 23.Caregiver training & 53.8 & 43.3 & 43.9 & 38.6 & 40.2 & 3.91 & 0.271 \\
\hline
\end{tabular}




\begin{tabular}{|c|c|c|c|c|c|c|c|}
\hline 24.Respite care & 44.0 & 31.8 & 35.3 & 33.5 & 33.9 & 1.45 & 0.694 \\
\hline $\begin{array}{l}\text { 25.ADL training and } \\
\text { prevention } \\
\text { anti-lost }\end{array}$ & 56.2 & 49.3 & 55.9 & 49.7 & 51.0 & 2.71 & 0.439 \\
\hline $\begin{array}{l}\text { 26.Medical equipment } \\
\text { purchase, rental, and } \\
\text { usage guide }\end{array}$ & 24.0 & 11.5 & 14.9 & 19.0 & 18.0 & 3.84 & 0.279 \\
\hline $\begin{array}{l}\text { 27.Hazard prevention } \\
\text { and self-rescue }\end{array}$ & 25.0 & 22.6 & 26.4 & 25.9 & 25.7 & 0.37 & 0.947 \\
\hline $\begin{array}{l}\text { 28.Legal inquiry } \\
\text { assistance }\end{array}$ & 29.2 & 20.3 & 23.3 & 23.2 & 23.2 & 0.78 & 0.854 \\
\hline $\begin{array}{l}\text { 29.Pressure ulcer } \\
\text { prevention }\end{array}$ & 61.3 & 58.6 & 60.2 & 60.5 & 60.4 & 0.12 & 0.990 \\
\hline 30.Pairing assistance & 28.6 & 30.9 & 32.9 & 26.0 & 27.6 & 3.56 & 0.313 \\
\hline
\end{tabular}

\subsection{Time spent on care services for PLWD}

Table 4 shows that were the intensity of service (hours per week) for each type of care. The General Care Services (ten items) were included in ECSP for PLWD have a total care service time of 34.95 hours per week, that is, 151.47 hours per month. The service time for community daycare, mental health counseling, and legal assistance have a significant difference with the levels of cognitive impairment $(\mathrm{P}<0.05)$.

Table 4. Intensity (hours per week) of services in care institutions, Mean (SD)

\begin{tabular}{|c|c|c|c|c|c|c|c|}
\hline \multirow[b]{2}{*}{ Services } & \multicolumn{5}{|c|}{ MMSE } & \multirow[b]{2}{*}{$\mathrm{F}$} & \multirow[b]{2}{*}{ P-value } \\
\hline & $\begin{array}{l}\text { Normal } \\
(\mathrm{n}=44)\end{array}$ & $\begin{array}{l}\text { Mild } \\
(\mathrm{n}=88)\end{array}$ & $\begin{array}{l}\text { Moderate } \\
(\mathrm{n}=213)\end{array}$ & $\begin{array}{l}\text { Severe } \\
(\mathrm{n}=954)\end{array}$ & $\begin{array}{c}\text { Total } \\
(\mathrm{n}=1299)\end{array}$ & & \\
\hline 1.Housekeeping & $8.0(2.7)$ & $6.6(2.5)$ & $7.5(2.7)$ & $7.0(2.7)$ & $7.1(2.6)$ & 0.30 & 0.827 \\
\hline 2.Transportation & $2.7(3.8)$ & $7.5(2.8)$ & $4.8(3.0)$ & $4.7(2.3)$ & $4.7(2.6)$ & 2.09 & 0.103 \\
\hline 3.Dining & $8.8(2.2)$ & $7.2(2.5)$ & $6.0(2.4)$ & $6.1(2.4)$ & $6.1(2.4)$ & 1.12 & 0.342 \\
\hline 4.Dressing/Grooming & $5.4(2.2)$ & $3.9(2.0)$ & $3.8(2.2)$ & $3.7(2.1)$ & $3.7(2.13)$ & 1.17 & 0.321 \\
\hline 5.Using the toilet & $4.7(1.9)$ & $4.1(2.5)$ & $4.5(2.4)$ & $4.3(2.2)$ & $4.3(2.3)$ & 0.17 & 0.920 \\
\hline $\begin{array}{l}\text { 6.Home } \\
\text { safety/modifications }\end{array}$ & $3.4(2.4)$ & $2.8(2.3)$ & $4.1(2.0)$ & $3.7(2.0)$ & $3.7(2.0)$ & 2.02 & 0.110 \\
\hline $\begin{array}{l}\text { 7.Community day } \\
\text { care }\end{array}$ & $27.3(1.9)$ & $31.7(1.9)$ & $28.7(2.3)$ & $14.3(3.0)$ & $18.1(2.9)$ & 9.00 & 0.000 \\
\hline $\begin{array}{l}\text { 8.Medical } \\
\text { records/Care plan }\end{array}$ & $2.0(2.3)$ & $1.7(2.6)$ & $2.0(2.3)$ & $2.2(2.3)$ & $2.1(2.3)$ & 1.49 & 0.216 \\
\hline 9.Primary care visit & $1.4(2.0)$ & $2.0(2.3)$ & $1.9(2.1)$ & $2.1(2.2)$ & $2.0(2.2)$ & 2.01 & 0.111 \\
\hline $\begin{array}{l}\text { 10.Regular health } \\
\text { evaluation }\end{array}$ & $1.7(2.4)$ & $1.7(2.6)$ & $2.0(2.4)$ & $2.2(2.3)$ & $2.1(2.3)$ & 1.53 & 0.207 \\
\hline $\begin{array}{l}\text { 11.Medication } \\
\text { management }\end{array}$ & $1.5(2.4)$ & $1.8(2.0)$ & $1.7(2.1)$ & $1.9(2.2)$ & $1.9(2.2)$ & 1.70 & 0.165 \\
\hline 12.Expectoration care & $1.2(2.9)$ & $2.2(2.5)$ & $1.9(2.1)$ & $1.7(2.1)$ & $1.8(2.2)$ & 2.20 & 0.088 \\
\hline 13.Oral care & $1.9(3.1)$ & $1.8(2.2)$ & $1.9(2.2)$ & $2.0(2.3)$ & $2.0(2.3)$ & 0.45 & 0.716 \\
\hline
\end{tabular}




\begin{tabular}{|c|c|c|c|c|c|c|c|}
\hline 14.Pain management & $2.7(2.9)$ & $2.6(2.3)$ & $2.5(2.2)$ & $3.0(2.5)$ & $2.9(2.5)$ & 0.64 & 0.587 \\
\hline $\begin{array}{l}\text { 15.Emergency } \\
\text { assistance }\end{array}$ & $1.9(2.7)$ & $1.4(2.1)$ & $1.5(2.1)$ & $1.9(2.1)$ & $1.8(2.1)$ & 2.44 & 0.065 \\
\hline $\begin{array}{l}\text { 16.Specialty nursing } \\
\text { care }\end{array}$ & $2.4(2.9)$ & $3.8(2.8)$ & $3.5(2.8)$ & $3.5(2.6)$ & $3.5(2.6)$ & 0.67 & 0.574 \\
\hline 17.Family guest beds & $2.5(3.5)$ & $2.7(3.2)$ & $3.6(2.9)$ & $4.3(2.9)$ & $4.0(2.9)$ & 1.59 & 0.192 \\
\hline 18.Companion & $3.0(2.5)$ & $4.7(2.4)$ & $4.0(2.7)$ & $4.9(2.5)$ & $4.7(2.5)$ & 2.52 & 0.057 \\
\hline 19.Life enrichment & $3.1(2.5)$ & $5.3(2.2)$ & $4.4(2.5)$ & $4.3(2.3)$ & $4.3(2.3)$ & 1.28 & 0.280 \\
\hline 20.Healthcare & & & & & & & \\
\hline $\begin{array}{l}\text { consultation and } \\
\text { education }\end{array}$ & $1.9(2.3)$ & $2.4(2.6)$ & $2.1(2.4)$ & $2.3(2.3)$ & $2.3(2.4)$ & 0.73 & 0.534 \\
\hline $\begin{array}{l}\text { 21.Mental health } \\
\text { counseling }\end{array}$ & $2.9(2.2)$ & $3.8(2.5)$ & $2.8(2.4)$ & $3.9(2.3)$ & $3.6(2.3)$ & 3.75 & 0.011 \\
\hline 22.Hospice care & $1.5(1.6)$ & $2.1(2.1)$ & $1.9(1.9)$ & $2.0(1.7)$ & $2.0(1.8)$ & 0.95 & 0.418 \\
\hline 23.Caregiver training & $2.1(2.5)$ & $3.0(2.4)$ & $2.6(2.2)$ & $2.9(2.1)$ & $2.8(2.1)$ & 1.16 & 0.326 \\
\hline 24.Respite care & $2.1(2.4)$ & $3.5(2.8)$ & $2.4(2.4)$ & $3.2(2.3)$ & $3.0(2.4)$ & 1.89 & 0.132 \\
\hline $\begin{array}{l}\text { 25.ADL training and } \\
\text { prevention } \\
\text { anti-lost }\end{array}$ & $5.3(3.5)$ & $6.5(1.6)$ & $5.9(2.3)$ & $5.5(2.2)$ & $5.6(2.3)$ & 0.41 & 0.748 \\
\hline $\begin{array}{l}\text { 26.Medical equipment } \\
\text { purchase, rental, and } \\
\text { usage guide }\end{array}$ & $2.2(2.6)$ & $2.1(2.4)$ & $2.1(2.6)$ & $2.4(2.2)$ & $2.3(2.3)$ & 0.40 & 0.752 \\
\hline $\begin{array}{l}\text { 27.Hazard prevention } \\
\text { and self-rescue }\end{array}$ & $1.6(2.6)$ & $1.7(2.2)$ & $1.7(1.9)$ & $2.2(2.2)$ & $2.0(2.2)$ & 1.96 & 0.120 \\
\hline $\begin{array}{l}\text { 28.Legal inquiry } \\
\text { assistance }\end{array}$ & $2.2(1.9)$ & $1.9(1.7)$ & $1.6(1.7)$ & $2.3(1.8)$ & $2.1(1.8)$ & 2.81 & 0.041 \\
\hline $\begin{array}{l}\text { 29.Pressure ulcer } \\
\text { prevention }\end{array}$ & $2.7(3.1)$ & $2.6(2.9)$ & $2.8(2.3)$ & $3.5(2.7)$ & $3.3(2.6)$ & 2.08 & 0.103 \\
\hline 30.Pairing assistance & $2.6(2.4)$ & $4.2(2.6)$ & $3.1(2.5)$ & $3.3(2.2)$ & $3.3(2.3)$ & 0.74 & 0.529 \\
\hline
\end{tabular}

3.6. The monthly standard base rate for ECSP

Based on $30 \%, 40 \%$, and $50 \%$ of the average monthly wage (1037.83 USD) of all urban units in China in 2018[31], the monthly standard base rate for ECSP nursing services at low-, medium, and high levels are in 2018 were calculated at 311.35 USD per month, 415.13 USD per month and 518.92 USD per month, respectively.

3.6.1 Calculation of monthly service hours

(1)Total Service Time Spent on Low-Level Care: The total time of care service for the General Care Services (ten items) was included in ECSP for PLWD with low-level care is 151.47 hours per month.

(2)Total Service Time Spent on Mid-Level Care $=151.47 \times \frac{415.13}{311.35}=201.96$ (hours per month)

(3)Total Service Time Spent on High-Level Care: $=151.47 \times \frac{518.92}{311.35}=252.45$ (hours per month) 


\subsubsection{Calculation of total number of service items}

(1) The number of basic care services for the low-level PLWD: it is the numbers of General Care Services were included in ECSP for PLWD, with a total of ten items of basic care services.

(2) The total number of mid-level care service items:

$=10 \times \frac{201.95}{151.47}=13.3 \approx 13$ (items)

(3) The total number of high-level care service items:

$=10 \times \frac{252.45}{151.47}=16.7 \approx 17$ (items)

\subsubsection{Selection of items of ECSP at different care levels}

For mild, moderate, and severe dementia, the ECSP corresponds to the low, medium, and high levels of care. The selection plan for ECSP at different levels is designed as "General Care Services + Elective Care Services", in which the service items for low-level, mid-level, and high-level care with the cognition severity of low, moderate, and severe for PLWD are 7+3,7+6, and 7+10, respectively (Table 5).

Table 5. Selection plan for service items on different care levels.

\begin{tabular}{lccc}
\hline \multicolumn{1}{c}{ Services } & Low & Midium & High \\
\hline Total number of care service items & 10 & 13 & 17 \\
Number of General Care Services items & 7 & 7 & 7 \\
Number of Selective Care Services items & 3 & 6 & 10 \\
Monthly care service time (hours/month) & 151.47 & 201.96 & 252.45 \\
\hline
\end{tabular}

\section{Discussion}

4.1. The ECSP for PLWD is a practicable and effective quantitative management means to promote the implementation of the long-term care insurance system

There is a paucity of research on estimating levels of care and ECSP for the growing PLWD population in mainland China. Due to the rapid population aging, the challenges of financing and service provision for PLWD requires proactive planning and support from individuals, families, and national and local governments. Dementia is one of the most important variables in LTCI for disabled elderly. To the best of our knowledge, the present study for first time quantified the substantial perceived needs and actual utilization from a survey of PLWD in six cities in central and eastern China. An ECSP consists of thirty care service items would be sufficient to ensure adequate service for this population. These findings provide an important first step to inform clinicians and policymakers regarding the design of LTCI coverage for the financing and delivery of PLWD care.

ESP (or "basic") refers to a basket of health services for government programs to finance health services in low- and middle-income countries [32]. Cost-benefit analysis of health benefit maximization was used as the gold standard is the key to achieving universal healthcare access (UHC). Many developed countries have adopted such a framework in guiding their long-term care financing, including those for the PLWD population. For example, Australia has developed home and community care service packages for the elderly at four levels of care needs (basic, low, moderate, and high), of which the corresponding highest level of government subsidy costs in July 2019 was 8,750 AUD, 15,250 AUD, 33,500 AUD, and 50,750 AUD, respectively [33]. In Japan, seven levels of care system based on ADL and the supportive care needs, with the government covering $50 \%$ of the total costs [34]. The US currently does not has an ECSP, but the development of a national plan for Alzheimer's disease and other related diseases is enacted by the Alzheimer's Disease Reporting Act of 2011 to improve care services and promoted the use of the Toolkit of Dementia Friendly Community, which includes the four stages of 
convening, engage, analyze, act, as well as its further detailed 17 implementation steps [35].

Researchers in China have tried to address issues of the ESP in health services and public policy research [36,37]. Ying et al. constructed a home care service package for the elderly with disabilities in Zhengzhou city and proposed the home care service packages into 11, 19, and 20 items for the elderly with mild, moderate, and severe disabilities, respectively [38]. Shanghai has stipulated 42 specific service items of community home care and care for the elderly and determined the payment standard for specific care services according to different evaluation levels of service objects [39]. Chengdu clarified that the mentally retarded people who meet the payment conditions of LTCI after assessment of dementia and disability can enjoy the LTCI treatment. The payment standard is determined by the corresponding care level of the disability. The monthly quota base of the first-, second-and third-levels of care is $50 \%, 40 \%$, and $30 \%$ of the average monthly wage of the employed personnel in all units in Chengdu, respectively [30].

The ECSP for PLWD proposed in this study is based on the political, economic, social, cultural, and historical context of LTC in China. By using a public financing perspective, and following the principles of public welfare, fairness, feasibility, and accessibility of basic care services, the resulting service estimates meet the national policy guidance aiming at ensuring universal coverage of a core set of services for person-centered dementia care [40]. It takes the monthly average wage of employees in all urban units in China as the calculation basis of monthly subsidy quota standard of basic care services and provides LTC services in the form of hierarchical care service packages, which includes those who need a rate of care service indicators for the mentally retarded elderly is more than $80 \%$ of the UHC project (low-level care service project). At the same time, in combination with the actual utilization of service items, a utilization rate of more than $60 \%$ is also included in the General Care Services items of ECSP for PLWD with low-level care service, and the rest are included in the moderate- and high-level care service package options. The "General Care Services + Selective Care Services" is proposed the modular combination of care service items conforms to Maslow's hierarchy of needs. On the one hand, considering the general requirements of the basic care services for the majority of PLWD, and guarantees the fairness and affordable accessibility of the most basic care services needs of PLWD at different levels; on the other hand, it also considered the personalized and diverse needs of PLWD with different levels of care services, which enhances its applicability. With the continuous improvement of China's economic and social development and the government's financial investment, the content of ESP should be monitored and revised as necessary. It will also play an important role in clarifying the main responsibility of the government in achieving the equalization of basic health and elderly care services [27]. Therefore, it is a practicable and effective quantitative management means to promote the implementation of the LTCI system for PLWD, and is also a useful tool for holding government, providers, and insurers accountable. It is deserved to an application on a large scale.

\subsection{Establish dementia care needs assessment and tiered service packages system}

A professional evaluation system for assessing the care service needs and classification service for PLWD should be established as an important part of the national LTCI program. This system will enable the linkage of care needs, service provision, and payment function that is standardized across the nation. For the elderly people with mild, moderate, and severe dementia who have been professionally assessed, they are provided with corresponding levels of care by community health service centers/hospitals, daycare centers, professional care institutions, tertiary specialized hospitals, and other institutions with personalized service offerings. Some safeguard mechanisms and operational implementing pathways of ECSP for PLWD in China are proposed, which are to make dementia a public health and social care priority, increase investment in infrastructure, incorporate the ECSP into the LTCI system, strengthen the care integration of 
community resources and care network for dementia, constantly promote the function of professional care institutions, community, and home care services, improve the cost-effectiveness ratio of ECSP for PLWD and improve the utilization efficiency [41].

\subsection{Strengths and limitations}

Our study has several strengths including large-scale sample data on service needs and utilization of the PLWD population in mainland China, as well as a well-validated electronic medical record system is used to identify the diagnosis and classification of dementia. Data from the National Bureau of Statistics of China represented the most authoritative sources for wage data used to estimate care costs. The study also provides a richer body of information on the ECSP and a clear description of what services will be available for every PLWD that is essential for health policy analysis and formulation as well as the basis for future planning of the LTCI system in China.

Several limitations should be considered. In general, there were various care needs and utilization rates during different stages of dementia. Due to the more notable disability and impaired functional status of the institutionalized patients, they are more severe clinical features among institutionalized patients than patients living at home. As these participants are all institutionalized individuals in our study, findings may not represent PLWD at mild to moderate stages of the disease. Future larger scale studies covering more geographic locations should be conducted to improve the accuracy of the estimates.

\section{Conclusions}

The present study represents one of the first large scale efforts to develop a need-based ECSP for PLWD in mainland China. Findings of this study are expected to provide important information for policymakers, clinicians, and researchers in establish an effective LTCI for PLWD, building a nationwide dementia action plan to develop an integrated health and social care system in China and other low/middle-income countries.

Author Contributions: Conceptualization, Tongda Sun, Hongdao Meng and Rangcheng Jia; Data curation, Tongda Sun, Hongdao Meng, Mingze Zhu, Naidan Tu and Rangcheng Jia; Formal analysis, Tongda Sun, Hongdao Meng, Mingze Zhu and Naidan Tu; Funding acquisition, Rangcheng Jia; Investigation, Tongda Sun, Hongdao Meng, Xiaoxin Dong, Ning Sun and Rangcheng Jia; Methodology, Tongda Sun and Hongdao Meng; Project administration, Tongda Sun and Rangcheng Jia; Resources, Tongda Sun, Hongdao Meng, Mingze Zhu, Xiaoxin Dong, Naidan Tu and Ning Sun; Software, Tongda Sun and Hongdao Meng; Supervision, Rangcheng Jia; Validation, Rangcheng Jia; Visualization, Hongdao Meng; Writing - original draft, Tongda Sun, Hongdao Meng, Mingze Zhu, Naidan Tu and Rangcheng Jia; Writing - review \& editing, Tongda Sun, Hongdao Meng and Rangcheng Jia. All authors have read and agreed to the published version of the manuscript.

Funding: This research was funded by the National Natural Science Foundation of China (Project number: 71774092) and The APC was funded by the National Natural Science Foundation of China in this section.

Institutional Review Board Statement: All subjects gave their informed consent for inclusion before they participated in the study. The study was conducted following the Declaration of Helsinki, and the protocol was approved by the Ethics Committee of Ningbo College of Health Sciences (Approval No. NBWY-011).

Informed Consent Statement: Informed consent was obtained from all subjects involved in the study. The caregivers or legal guardians of the participants were informed about the aims of the study and the extent of their participation before they gave written informed consent.

Data Availability Statement: The data presented in this study are available on request from the corresponding author. The data are not publicly available due to ethical restrictions and patient confidentiality. 


\begin{abstract}
Acknowledgments: We thank all of the participants who were enrolled in the study including all investigators and interviewers contributing to the data collection. We are grateful to Dr. Hansheng Ding, from Center for Health Development Research of Shanghai City, China; and Dr. Yongxue Yang, from Geriatric Hospital of Chengdu City, China, for their technical and invaluable assistance in the development of the questionnaire and operating procedures.
\end{abstract}

Conflicts of Interest: The authors declare no conflict of interests.

\title{
References
}

1. Alzheimer's Disease International (ADI). World Alzheimer Report 2015. London: Alzheimer's Disease International, 2015.

2. Alzheimer's Disease International (ADI). World Alzheimer Report 2016. London: Alzheimer's Disease International, 2016.

3. World Health Organization (WHO). Dementia: a public health priority. Geneva: World Health Organization, 2012.

4. Xu J, Wang J, Wimo A, Fratiglioi L, Qiu C. The economic burden of dementia in China, 1990-2030: implications for health policy. Bull World Health Organ. 2017, 95,18-26.

5. Jianping Jia, Cuibai Wei, Shuoqi Chen, Fangyu Li, Yi Tang, Wei Qin, Lina Zhao, et al. The cost of Alzheimer's disease in China and re-estimation of costs worldwide. Alzheimer's \& Dementia. 2018, 14, 483-491.

6. Yang W, He AJ, Fang L, Mossialos E. Financing institutional long-term care for the elderly in China: a policy evaluation of new models. Health Policy and Planning. 2016, 31, 1391-1401.

7. Lu B, Mi H, Zhu Y, Piggott J. A sustainable long-term health care system for aging China: a case study of regional practice. Health Systems \& Reform. 2017, 3, 182-190.

8. Peng Du, Lingyao Ji. Progress, Challenges and prospects of long-term care policy in China. Chinese Journal of Health Policy. 2019, 12, 29-34. (in Chinese).

9. Shiying He, Ruiming Dai, Ying Wang, Man Jiang, Ge Bai, Li Luo. Comparative research of the financing scheme for long-term care insurance in pilot areas in China. Chinese Health Resources. 2019, 22, 28-34. (in Chinese).

10. Guibin Yang, Hongzhong Xie. A comparative analysis of pilot services of long-term care insurance system in China-based on the comparison between Shanghai, Nantong and Qingdao. J Xi'an Univ of Arch \& Tech (Social Science Edition). 2020, 39, 35-41. (in Chinese).

11. Mosca I, van der Wees PJ, Mot ES, Wammes JJ, Jeurissen PP. Sustainability of long-term care: puzzling tasks ahead for policy-makers. Int J Health Policy Manag. 2017, 6, 195-205.

12. Wang S, Cheung DSK, Leung AYM. Overview of dementia care under the three-tier long-term care system of China. Public Health Nurs. 2019, 36, 199-206.

13. General Office of Ministry of Human Resources and Social Security. The guidance for carrying out the pilot of long term care insurance system. (Jun. 27, 2016). Available at http://www.gov.cn/xinwen/2016-07/08/content_5089283.htm. Accessed on Sep.8, 2020. (in Chinese).

14. National Healthcare Security Administration, Ministry of Finance. The guidance for extending to carry out the pilot of long term care insurance system. (Sep. 10, 2020). Available at http://www.gov.cn/zhengce/zhengceku/2020-11/05/content_5557630.htm. Accessed on Dec.8, 2020. (in Chinese).

15. Lu Shu, Qun Wang. Research on the Long-term Care Insurance Security Policy of the Demented Elderly in China. Health Economic Research. 2020, 37, 7-10. (in Chinese).

16. Longfei Jia, Meina Quan, Yue Fu, Tan Zhao, Yan Li, Cuibai Wei, Yi Tang, Qi Qin, Fen Wang, Yuchen Qiao, Shengliang Shi, Yan-Jiang Wang, Yifeng Du, Jiewen Zhang, Junjian Zhang, Benyan Luo, Qiumin Qu, Chunkui Zhou, Serge Gauthier, Jianping Jia, Group for the Project of Dementia Situation in China. Dementia in China: epidemiology, clinical management, and research advances. Lancet Neurol. 2020, 19, 81-92.

17. World Health Organization (WHO). International classification of disease, 10th revision (ICD-10). Geneva; World Health Organization. 1999.

18. American Psychiatric Association. Diagnostic and Statistical Manual of Mental Disorders, 4th edn. Washington DC: American Psychiatric Association. 1994.

19. American Psychiatric Association. Diagnostic and Statistical Manual of Mental Disorders, 5th edn. Arlington, VA: American Psychiatric Publishing. 2013.

20. Jia J. Chinese guideline for dementia and cognitive disorders. Beijing: People's Medical Publishing House, 2016. (in Chinese).

21. Jia J. Chinese guideline for diagnosis and treatment of dementia and cognitive disorders: diagnostic criteria of dementia and its subtypes. Natl Med J China, 2018, 98, 965-970. (in Chinese).

22. Collin C,Wade DT, Davies S, Horne V. The Barthel ADL Index: a reliability study. Int Disabil Studies.1988, 10, 61-63.

23. Shujuan Liu. Long Term Care. Taiwan: Huaxing Publishing Co. LTD. 2nd. 2011. (in Chinese).

24. M F Folstein, S E Folstein, P R McHugh. "Mini-mental State". A Practical Method for Grading the Cognitive State of Patients for the Clinician. J Psychiatr Res. 1975, 12, 189-98.

25. Xin Ying Chua, Robin Wai Munn Choo, Ngoc Huong Lien Ha, Chin Yee Cheong, Shiou Liang Wee, Philip Lin Kiat Yap. Mapping Modified Mini-Mental State Examination (MMSE) Scores to Dementia Stages in a Multi-Ethnic Asian Population. Int Psychogeriatr. 2019, 31, 147-151.

26. Fadi El-Jardali, RachaFadlallah, ArefDaouk, RanaRizk, NourHemadi, Ola El Kebbi, Aida Farha, Elie A Akl. Barriers and Facilitators to Implementation of Essential Health Benefits Package Within Primary Health Care Settings in Low-Income and Middle-Income Countries: A Systematic Review. Int J Health Plan Manage. 2019, 34, 15-41.

27. Tongda Sun, Yitun Sun, Xiaoxin Dong, Rangcheng Jia. Discussion on the Conceptual Characteristics and Realization Path of the Basic Care Service Package for the Demented Elderly. Health Economic Research. 2019, 36, 6-8. (in Chinese).

28. Ministry of Labour, Ministry of Finance, All-China Federation of Trade Unions. Advise on the Rate-adjusting policy of nursing subsidy for disablement employees caused by job. Sep. 29, 1992. (in Chinese).

29. Minglai Zhu, Qingxian Jia. The analysis of demand for long-term care and its insurance system constructing in China. Chinese Journal of Health Policy. 2009, 2, 32-38. (in Chinese). 
30. Human Resources and Social Security Bureau of Chengdu City, Bureau of Finance of Chengdu City. Advise on the implementation rules of long term care insurance in Chengdu, China (Demonstration). Available at http://www.cdhrss.gov.cn/cdrsj/c109727/2019-01/08/content_9be6a0e77e8f4541a71f065332f66a94.shtml. (Oct. 10, 2018). Accessed on Jan. 22, 2019. (in Chinese).

31. National Bureau of Statistics of China. China statistical yearbook 2019. Available at http://www.stats.gov.cn/tjsj/ndsj/2019/indexch.htm. Accessed on Sep. 9, 2020. (in Chinese).

32. World Bank. Investing in health: world development report 2003. New York: Oxford University Press. 2003.

33. Australian Government Department of Health. The Department of Health Your guide to Home Care Package services. (2019-11-25). Available at https://agedcare.health.gov.au/programs/my-aged-care. Accessed on Jan. 10, 2020.

34. Rhee JC, Done N, Anderson GF. Considering long-term care insurance for middle-income countries: comparing South Korea with Japan and Germany. Health Policy. 2015, 119, 1319-1329.

35. Dementia Friendly America ${ }^{\circledR}$ (DFA). Dementia Friendly Communities Toolkit. Available at https://actonalz.org/dementia-friendly-toolkit. Accessed on Aug.10, 2020.

36. Anning, Ma, Wengui Zheng, Peicheng Wang, Hongcai Zhang, Haiming Guo, Hongqi Sheng, Chunxiang Wang, Weiqin Chai. Overview of basic public health service package of the nation. Health Economic Research. 2008, 4, 8-10. (in Chinese).

37. Hanping Jiang, Yuanqing Chen, Lexuan Luo, Shaoxian Chen, Xia Chen, Xipo Li, Yixiang Huang. Thought design of cost estimate on community public health service pack in Shenzhen city. Chinese Hospital Management. 2010, 30, 10-12. (in Chinese).

38. Ying Chen, Lixia Ma, Huili Pei, Lingling Liu, Zhenxiang Zhang. Home-based Care Service Package for urban disabled elderly in Zhengzhou, China. Chin J Rehabil Theory Pract. 2017, 23, 118-122. (in Chinese).

39. Shanghai Municipal Peoples Government. Advise on the implementation rules of pilot program of long-term nursing insurance in Shanghai, China (Demonstration). (Dec.29, 2016). Available at http://www.shanghai.gov.cn/nw2/nw2314/nw2319/nw12344/u26aw51124.html. Accessed on Sep. 8, 2020. (in Chinese).

40. de Blok C, Luijkx K, Meijboom B, Schols J. Improving long-term care provision: towards demand-based care by means of modularity. BMC Health Services Research. 2010. 10:278.

41. World Health Organization (WHO). Global action plan on the public health response to dementia 2017-2025. Geneva: World Health Organization, 2017. 